\title{
电场作用下水表面电势的分子动力学研究
}

\author{
杨鹏里 $^{a}$ 王振兴 ${ }^{b} \quad$ 梁尊 $^{a} \quad$ 梁洪涛 ${ }^{a}$ 杨洋*,a \\ ( $a$ 华东师范大学 物理与材料科学学院 凝聚态物理研究所 上海 200241) \\ ( 微宏动力系统(湖州)有限公司 湖州 313000)
}

\begin{abstract}
摘要 水表面电势在诸多电化学过程与反应中扮演关键角色, 然而实验上直接测量却极具挑战. 本论文提出一套基于 平衡态恒定电势分子动力学的模拟一分析-计算方法, 可实现通过保持恒定电势且伴随电荷涨落的电极板将电场作用于 附近的水表面，并以平均探针电势计算方法精确测量空间电势分布. 凭借此套方法，首次计算了不同电极电势下水表 面区域的空间电势分布函数，并测得了鲜有报道的水表面电势随外电场的变化关系. 发现了阴极附近水的表面电势随 外电场增强而降低而阳极附近水的表面电势随外电场增强而增大的非对称性. 同时计算了平衡态水表面分子数密度和 偶极矩极化密度分布函数, 展示出逐渐增强的外电场能够强烈改变水表面区域的极化行为也能够使液体水整体微弱的 极化. 论文最后提出水表面电势随电场变化的非对称性源自水表面极化行为的非对称性以及液体区域的整体极化. 关键词＼cjkstart水; 分子动力学; 恒定电势法; 液-汽界面; 平均探针电势计算法
\end{abstract}

\section{A Molecular Dynamics Simulation Study of the Effect of External Electric Field on the Water Surface Potential}

\author{
Yang, Pengli ${ }^{a} \quad$ Wang, Zhenxing ${ }^{b} \quad$ Liang, Zun $^{a} \quad$ Liang, Hongtao $^{a} \quad$ Yang, Yang ${ }^{*, a}$ \\ $\left({ }^{a}\right.$ School of Physics and Material Science, East China Normal University, Shanghai 200241) \\ ( ${ }^{b}$ Microvast (Huzhou) Co. Ltd., Huzhou, 313000)
}

\begin{abstract}
The surface potential of the liquid-vapor interface of water plays a critical role in electrochemistry, interfacial reactivity, and solvation thermodynamics. However, direct experimental measurement of the surface potential of pure water is exceedingly challenging. Here we present a methodology to explore the effect of external electric field on the water surface potential. The methodology contains constant electrostatic potential molecular dynamics simulation [J. Chem. Phys., 126, 084704(2007)], in which, the electrode charges are allowed to fluctuate to keep the potential fixed, as well as a recently developed probe and average method [J. Phys.: Cond. Matter, 28, 464006(2016)] to accurately map out the electrostatic potential across the water surfaces. The methodology is applied to the coexistence of the vapor phase and the liquid phase of the room temperature pure water (described by a simple SPC/E water model) under different magnitudes of E-fields generated from the nearby electrodes, yielding a first-time calculation of the external E-field dependent water surface potential profiles, and the relationship between the water surface potential and the external E-field strength which has been rarely reported. We found an asymmetric effect of external E-field on the surface potential, i.e., the surface potential decreases with increasing the external E-field strength for the water surface close to the cathode, while the surface potential increases with increasing field strength for the surface close to the anode. The water surfaces are also characterized by calculating the number density and dipole polarization density profiles, which depict the presence of the external E-fields induced bulk polarization under high strength field. By comparing the dipole polarization density profiles and the potential profiles, we conclude that the asymmetric effect of external E-field on the surface potential is due to the asymmetric behavior in surface polarization under external E-field for the water surfaces near cathode or anode, and is also due to the polarization within bulk part of the liquid water. The methodology presented in the current study can be easily applied to more advanced water models such as polarizable water models which are beyond the SPC/E used in current work. The achievement of the fundamental data and the physics relationship between the surface potential of water and the applied external E-field could potentially facilitate the advancements in electrodynamics and thermodynamics of the liquid-vapor interfaces.
\end{abstract}

Keywords water; molecular dynamics; constant potential method; liquid-vapor interface; probe and average potential calculation method

\footnotetext{
*E-mail: yyang@phy.ecnu.edu.cn

Received June 10, 2019; published August 28, 2019.

Project supported by the National Natural Science Foundation of China (Nos. 11504110, 11874147), the Science and Technology Project of Shanghai Science and Technology Commission (No. 18DZ1112700), the Fundamental Research Funds for the Central Universities, and the East China Normal University Multifunctional Platform for Innovation (001).

项目受国家自然科学基金(Nos. 11504110, 11874147)、上海市科委科技项目(No. 18DZ1112700)、中央高校基本科研业务费专项资金资助和华东师范 大学公共创新服务平台(001)资助.
} 


\section{1 引言}

由于水分子自身较强的极性, 电场作用下的液态水 或固态冰常展现具有高应用价值的新奇现象和过程. 如 电润湿现象 ${ }^{[1,2]}$ 、电场驱动的射流 ${ }^{[3]}$; 又如冰在水中的形 核 ${ }^{[4 ~ 8]}$ 、冰的电熔化 ${ }^{[9 \sim 11]}$ 、界面水的电凝固 ${ }^{[12 \sim 17]}$ 、电场 诱导的低维冰的铁电转变等 ${ }^{[18]}$ 与水相关的一系列相变 过程中, 相变势垒和相变速率可被外电场干预或调控. 因此，对电场作用下的水(或冰)的结构和热力学性质的 研究, 成为了国内外在过去几十年一个前沿热点 ${ }^{[19 ~ 24]}$. 目前, 这个热点领域一个关键任务就是研究清楚水的表 面性质在电场作用下的变化规律. 基于经典密度泛函理 论和 Gibbs 界面热力学的理论研究 ${ }^{[25,26]}$ 能预言出外电场 下简单极化分子体系表面过剩极化和表面张力的变化 规律. 但由于直接实验观测极具挑战, 电场作用下水表 面性质变化的实验结果数量圆乏且存在争议 ${ }^{[27 \sim 29]}$.

随着近年来水科学领域计算模拟的兴起 ${ }^{[30]}$, 本领 域中存在争议的问题以及实验数据稀缺的现状有望获 得改善. 国际上已有几个课题小组报道了关于空间匀强 电场作用下偶极分子液-汽界面的计算模拟研究工

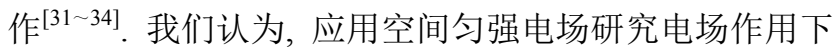
块体水或块体冰( “块体” 指应用周期性边界条件描述 的无限大体系)的结构与热力学行为是合理的 ${ }^{[19 \sim 24]}$, 但 在处理非匀质体系例如水的表面(液汽界面)时, 空间匀 强电场法则存在不足. 这是由于电场通过微观结构对称 破缺的极性材料界面物质时, 会产生偶极极化现象从而 导致局域电场被极化感生电场削弱, 而空间匀强电场下 体系每个带电荷粒子被强制赋予匀强电场带来的恒定 力, 不能准确重现表面极化屏蔽效应 ${ }^{[35,36]}$. 如要改进这 一方法上的不足, 需要更加合理的施加电场方式. 最近 由诺奖得主 Geim 教授课题组 ${ }^{[37]}$ 主导的水科学领域前沿 实验就是以原子力显微镜探针针尖作为电极, 对纳米尺 度的界面水/限域水施加电场来研究其介电性质. 由此 可见, 通过电极表面电荷施加外电场, 而非空间匀强电 场, 模拟会更加合理也更切合实际. 据我们知悉的文献 中, 尚未有将施加恒定电势的金属电极产生的电场作用 于附近水表面的模拟研究.

恒定电势分子动力学方法是一种能够实现金属电 极表面电荷对极性液体水密度涨落致使的电荷密度涨 落的动态响应并保持恒定电势的分子模拟方法. 这种方 法已在电极/电解液的界面体系研究中获得十分成功的 应用 ${ }^{[38 ~ 46]}$. 本论文的目标是在基于恒定电势分子动力 学方法模拟的电场作用水表面体系中测量水的表面电 势 $\chi$.

水的表面电势这一界面参量, 定义为水表面液相端 电势与汽相端电势的差(前者减后者 $)^{[47]}$, 在分析化学、 电化学、表面与胶体化学、大气化学、溶解热力学等诸 多分支学科中均具有重要研究价值 ${ }^{[48,49]}$. 它强烈影响界 面离子的分布、溶解过程的亲水/疏水行为和溶解自由能
的量值 ${ }^{[50 \sim 52]}$, 紧密联系着界面化学反应以及蛋白质或 磷脂膜导通正负电荷之间的差异性 ${ }^{[53]}$. 实验上测量水 的表面电势极其困难 ${ }^{[1,52]}$, 少量实验曾间接估测了常温 零外电场下水的表面电势, 这些实验估测的数据甚至连 正负号都不能统一 ${ }^{[54 ~ 57]}$. 我们认为水的表面电势是澄 清关于外电场作用下诸多水表面性质变化规律争议的 关键. 以外加电场的方向和强度做为控制变量来描述电 场作用下的水表面性质的变化规律, 是导致本领域诸多 争议的重要原因. 只有准确测量表面电势, 把其量值与 表面偶极极化等更深层微观结构/热力学性质的变化连 接起来, 才能抓住本质获得正确认识, 进而寻求定量调 控与应用. 然而目前为止, 水的表面电势研究仅限于零 外电场体系，尚未有任何关于电场作用下水表面电势变 化的实验测量或理论计算被报道过.

本论文中，提出一套基于平衡态分子动力学的模拟 与分析方法, 可实现将施加恒定电势的金属电极产生的 电场作用于附近水表面, 并能精准计算模拟体系的空间 电势分布，用以探索电场作用下水表面结构/热力学性 质变化规律. 应用此套方法, 我们模拟了 $\mathrm{SPC} / \mathrm{E}$ 模型描 述的室温水与金属电极板、真空层组成的平衡态系统, 计算了不同电极电势下水表面的密度与分子极化密度, 采用 Wang 等近期开发的平均探针方法(详见计算方法 部分)计算了模拟体系的空间电势分布和电场强度, 测 量出随外电场改变的表面电势、表面极化、表面宽度、 极化电场等一系列参数变化规律. 本论文提出的模拟与 计算方法能够为水表面科学提供重要数据, 对水系统的 液-汽两相平衡热力学理论发展具有重要意义.

\section{2 计算方法}

\section{1 恒定电势分子动力学方法}

经典的原子力场来描述导体非常困难, 所以应用分 子动力学模拟金属电极存在较大挑战. 在早期诸多金属 电极/电解液界面的分子动力学模拟工作中 ${ }^{[58 \sim 62]}$, 充电 电极板每个表面原子都被赋予相同数量的电荷. 这种恒 定电荷方法人为忽略了电极电荷对电解质局域电荷涨 落的响应. 由 Reed 等 ${ }^{[63,64]}$ 提出的恒定电势方法能够计 入系统中极性液体或带电离子溶液密度涨落诱导的极 板原子的电荷涨落变化. 在此恒定电势方法中, 分子动 力学演化的每一步, 都会改动每一个电极原子所带电荷 数量, 以满足每个电极原子所在位置的电势值为恒定数 值. 在这样的约束条件下, 整个电极上每个电极原子的 电势都恒等于预设的施加电势/电压 $\phi, \phi=\phi_{i}=\partial U / \partial q_{i}$, 其中 $i$ 是电极原子的序号, $U$ 是体系的总库伦势能. 恒定 电势方法的分子模拟实现大多基于 Gingrich 等 ${ }^{[65]}$ 开发 的高斯电荷替代点电荷的方法, 本论文中的恒定电势分 子动力学方法采用了 Wang 等 ${ }^{[6]}$ 在 2014 年提出的改进 版本，即对三维周期性 Ewald 求和并附加模拟体系周期 性盒子的形状进行修正, 最终获得的计算精度和计算速 
度均更出色.

对任意一个极板原子 $l$, 其对应的坐标位置为 $\mathbf{R}_{l}$, 电荷量为 $Q_{l}$, 该位置电势表示为 ${ }^{[66]}$ :

$$
\phi_{l}=\phi_{l}^{\text {real }}+\phi_{l}^{\mathrm{kspace}}+\phi_{l}^{\mathrm{self}}+\phi_{l}^{\mathrm{slab}}
$$

其中 $\phi_{l}^{\mathrm{real}}$ 是三维 Ewald 求和中实空间的组成部分,

$$
\begin{aligned}
\phi_{l}^{\mathrm{real}} & =\sum_{i=1}^{n^{\prime}} Q_{i} \frac{\operatorname{erfc}\left(\alpha\left|\mathbf{R}_{l}-\mathbf{R}_{i}\right|\right)-\operatorname{erfc}\left(\frac{\eta}{\sqrt{2}}\left|\mathbf{R}_{l}-\mathbf{R}_{i}\right|\right)}{\left|\mathbf{R}_{l}-\mathbf{R}_{i}\right|} \\
& +\sum_{j=1}^{m} q_{i} \frac{\operatorname{erfc}\left(\alpha\left|\mathbf{R}_{l}-\mathbf{r}_{j}\right|\right)-\operatorname{erfc}\left(\eta\left|\mathbf{R}_{l}-\mathbf{r}_{j}\right|\right)}{\left|\mathbf{R}_{l}-\mathbf{r}_{j}\right|}
\end{aligned}
$$

$n^{\prime}$ 指 $l$ 原子以外; $\phi_{l}^{\mathrm{kspace}}$ 是倒格矢空间组成部分,

$$
\phi_{l}^{\mathrm{kspace}}=\frac{1}{V} \sum_{\mathbf{k}>0} \Gamma(|\mathbf{k}|)\left[e^{-i \mathbf{k} \cdot \mathbf{R}_{l}} \boldsymbol{S}(\mathbf{k})+e^{i \mathbf{k} \cdot \mathbf{R}_{l}} \boldsymbol{S}(-\mathbf{k})\right]
$$

其中 $\mathbf{k}$ 是倒晶格, $\Gamma(|\mathbf{k}|)$ 是 Ewald 求和中波失依赖的傅 立叶系数, 结构因子

$$
\begin{aligned}
& \boldsymbol{S}(\mathbf{k})=\sum_{i=1}^{n} Q_{i} e^{i \mathbf{k} \cdot \mathbf{R}_{i}}+\sum_{j=1}^{m} q_{j} e^{i \mathbf{k} \cdot \mathbf{r}_{j}} \\
& \phi_{l}^{\text {self }}=\frac{\sqrt{2} \eta-2 \alpha}{\sqrt{\pi}} Q_{l}
\end{aligned}
$$

是自相互作用部分的修正项,

$$
\phi_{l}^{\mathrm{slab}}=\frac{4 \pi Z_{l}}{V}\left(\sum_{i=1}^{n} Q_{i} Z_{i}+\sum_{j=1}^{m} q_{j} z_{j}\right)
$$

是板状几何修正项, $Z_{i}$ 和 $z_{j}$ 表示极板和电解质原子 $z$ 分量 坐标. 综合上述分式, $\phi_{i}$ 可写成体系电荷的线性叠加,

$$
\phi_{l}=\sum_{i}^{n} a_{l i} Q_{i}+\sum_{j}^{m} b_{l j} q_{j}
$$

$a_{l i}$ 为极板原子位置坐标 $\mathbf{R}_{l}$ 与 $\mathbf{R}_{i}$ 的函数, 固定不变. 而 $b_{l j}$ 因电解质粒子时刻变化而需在分子模拟的每一步都 做更新. 模拟设定的恒定电势为 $V$, 极板每个原子都可 得到一个线性方程, 于是每一个时刻, 极板所有原子电 荷 $\mathbf{Q}=\left[Q_{1}, Q_{2}, Q_{3}, \ldots, Q_{n}\right]$ 可通过求解线性方程组 $\mathbf{A} \mathbf{Q}=V$ $-\mathbf{B}$ 获得.

\section{2 模拟方法}

模拟系统的构建基于直角坐标系. 分子动力学模拟 盒子包含两个面心立方晶格的平行金属铂电极板, 每个 铂电极中含 3 层原子, 处于 $300 \mathrm{~K}$ 温度下的金属铂面心 立方晶格(晶格常数 $0.39 \mathrm{~nm}$ ). 金属铂原子在整个模拟 过程中固定不动. 定义 $z$ 方向垂直电极板表面或水表面, 在 $x$ 和 $y$ 方向(即极板/水表面平行方向)上应用周期性边 界条件. $x, y$ 和 $z$ 三个方向上的尺寸分别为 5.25, 5.25 和 $14.5 \mathrm{~nm}$. 两平行极板间, 盒子 $z$ 坐标的正中放置一块 7 $\mathrm{nm}$ 厚的液体水膜(包含 6750 个水分子). 两个电极板的 内表面的位置位于 $z=-6.7 \mathrm{~nm}$ 和 $z=6.7 \mathrm{~nm}$, 电极的内 表面面向水膜的两个(液汽)表面. 原子力显微镜探索冰 表面准液相层的实验 ${ }^{[67]}$ 与分子模拟中 ${ }^{[68]}$, 针尖与水表 面在 $2 \mathrm{~nm}$ 的距离以内会因毛细作用连接起来, 因此, 本 研究选取电极内表面到相对的水表面的距离约为 $3 \mathrm{~nm}$.

我们在本研究中选用了一个最简单且流行的刚性 水分子模型, 即 $\mathrm{SPC} / \mathrm{E}$ 力场模型 ${ }^{[69]}$. 需要说明的是, 本 论文目的是提出新的分子动力学模拟方法, 因此水模型 的选取并不是核心问题。在这个水模型中，原子电荷和 质量都集中在同一个质点上, 氧原子电荷量为 $-0.8476 \mathrm{e}$, 氢原子电荷量为 $0.4238 \mathrm{e}$. 氧原子间取 Lennard-Jones $(\mathrm{LJ})$ 势相互作用 (具体 $\mathrm{LJ}$ 参数为 $\sigma_{\mathrm{OO}}=$ $\left.0.3166 \mathrm{~nm}, \varepsilon_{\mathrm{OO}}=1.0797 \times 10^{-21} \mathrm{~J}\right)$. 氢氧键的键长为 0.1 $\mathrm{nm}$, 氢氧氢键角为 $109.47^{\circ}$. 恒定电势方法被用来处理 金属铂电极铂原子上电荷的涨落, 我们使用了 Wang 等 开发的适用于(美国 Sandia 国家实验室开发的分子动力 学模拟软件)LAMMPS 的恒定电势法代码 ${ }^{[66]}$, 其中包括 应用 Particle-Particle-Particle-Mesh 算法处理长程静电相 互作用, 和在 $z$ 方向上引入一个长度约为 $50 \mathrm{~nm}$ 的真空 区域去除模拟盒子与镜像之间偶极相互作用 ${ }^{[70]}$.

本工作中所有的分子动力学模拟通过美国 Sandia 国家实验室开发的 LAMMPS 软件 ${ }^{[66]}$ 实现. 应用 SHAKE 算法来实现 $\mathrm{SPC} / \mathrm{E}$ 水分子运动的刚性约束条件 ${ }^{[71]}$, 每个 氢氧键键长和键角维持不变. 运动方程的积分使用 $N V T$ 正则系综(分子数 $N$ 、体积 $V$ 、温度 $T$ 恒定), 时间步长取 $1 \mathrm{fs}\left(10^{-15} \mathrm{~s}\right)$, 利用 Nosé-Hoover 控温法恒稳 $300 \mathrm{~K}$ 温度. 已有文献 ${ }^{[72]}$ 报道过 $316 \mathrm{~K}$ 温度下 $\mathrm{SPC} / \mathrm{E}$ 模型的液汽两相 平衡密度, 其中汽体密度 $0.0001 \mathrm{~g} / \mathrm{cm}^{3}$, 这意味着在我 们模拟体系中极板到水表面间的空间, 汽相水分子数目 应为 0 . 我们的模拟中确实发现了汽相水分子, 但出现 概率极低. 本工作共模拟了 4 组不同电极施加电势组合, 两个电极施加电势值分别为 $0 \mathrm{~V}$ 和 $0 \mathrm{~V},-1 \mathrm{~V}$ 和 $1 \mathrm{~V}$, $-3 \mathrm{~V}$ 和 $3 \mathrm{~V},-4.5 \mathrm{~V}$ 和 $4.5 \mathrm{~V}$. 两电极板电势差分别为 0 $\mathrm{V} 、 2 \mathrm{~V} 、 6 \mathrm{~V}$ 和 $9 \mathrm{~V}$. 我们在 $z$ 坐标为负的电极上施加负 电势, 定义其为阴极; 在 $z$ 坐标为正的电极上施加正电 势，定义其为阳极. 对每组体系先模拟 2000000 步，使 之达到结构、热力学和静电力学平衡, 之后 20000000 步模拟中每 200 步记录一次原子坐标信息, 用以后续统 计分析与计算.

\section{3 平均探针电势计算方法}

平均探针电势计算方法由 Wang 等 ${ }^{[73]}$ 于 2016 年提 出, 这个方法可直接获得 $x y z$ 三维空间网格上每一点的 电势. 在此网格上任意一点放置一个虚拟的探针 $k$, 其 在电场中的电势 $\phi_{k}$ 为:

$$
\phi_{k}=\sum_{i}^{n} \frac{q_{n}}{\left|\mathbf{r}_{k}-\mathbf{r}_{n}+n \boldsymbol{L}\right|}
$$


其中 $\mathbf{r}_{k}$ 是虚拟探针放置的网格位置, $\mathbf{r}_{n}$ 和 $q_{n}$ 是系统中任 意一个原子的坐标和其附带的电荷数量, $\mathrm{n} \boldsymbol{L}$ 表示模拟盒 子镜像平移矢量. 利用 Ewald 求和, 此方法能够在分子 动力学模拟的每一步都提供完整三维静电电势场. 对取 样时刻平均可获得系统平均静电电势场 $\phi(r)$. 对极板 法向( $z$ 轴)上任一位置所对应的 $x y$ 面所有探针格点平均, 可获得 $z$ 方向电势分布函数, $\phi(z)$. 实际计算中, 电势 空间分布的计算结果与探针密度有关, 为保证高精度, $z$ 方向上网格点间距取 $0.005 \mathrm{~nm}, x y$ 平面内放置 $50 \times 50$ 共 2500 个探针格点.

平均探针法以外的电势分布计算方法, 多基于数值 求解泊松方程:

$$
\nabla^{2} \phi=\rho(\boldsymbol{r}) / \varepsilon
$$

$\varepsilon$ 为真空介电常数, $\rho(\boldsymbol{r})$ 为电荷密度场. 为了简便, 具体 求解时常忽略平行极板方向上的电荷梯度而仅关心一 维电势分布, 于是 $z$ 方向上电势分布函数理论上就可用 电荷密度分布函数的双重积分获得. 简单数值积分方法 完全无法满足该双重积分的精度需求, 需用到包括有限 差分法 ${ }^{[74]}$ 、单边格林函数法 ${ }^{[75,76]}$ 和离散傅里叶变换法 ${ }^{[77]}$ 这三种较为高阶的求解方法. 可是无论从精度、积分收 敛度还是边界条件的依赖性上，上述积分方法摆脱不了 数值求解泊松方程的限制要求. 平均探针法的提出, 使 确定电势分布函数的时候无需数值积分、不依赖积分边 界条件, 获得的结果数值误差远小于上述三种积分方 法 ${ }^{[73]}$. 平均探针法还拥有三维空间的电势场可直接计 算的优点, 并非常容易拓展到任意几何形状的系统中.

\section{4 热力学参量分布函数计算}

我们计算一系列热力学参量分布函数用来定量表 征水的液汽表面重要结构与热力学性质的变化规律. 这 些参量包括电势、水分子数密度以及偶极矩极化密度. 这些分布函数描绘出目标参量沿 $z$ 方向的数值变化. 对 三维系统, 沿 $z$ 方向上进行细分, 获得宽度为 $0.005 \mathrm{~nm}$ 的细粒度 “切片” , 统计每个 “切片” 内目标参量平均 数值. 例如, 电势分布函数 $\phi(z)$ 通过计算每个切片中 2500 个探针格点的电势平均值获得; 水分子数密度 $\rho(z)$ 通过计算氧原子平均数与该切片体积的比值获得; 偶极 矩极化密度 $M_{z}(z)$ 通过计算切片中单位体积的平均偶极 矩 $(z$ 分量) 获得. 利用误差函数拟合水分子密度分布函 数我们可计算出的水表面位置 ${ }^{[78]}$ 和表面区域宽度 $\left(w_{1090}\right)$. 利用此表面位置信息, 我们从不同(电极施加电 势差)模拟体系的电势分布函数和偶极极化密度分布函 数中提取中水表面关键参量, 包括表面电势 $\chi$ 和表面极 化 $\mathcal{P}$ 等. 从而获得这些参量随电极施加电势变化规律, 并得到这些表面参量之间以及与水膜体电势之间的内 在关联.

\section{3 结果与讨论}

图 1(a)中记录了两电极板电势差 $2 \mathrm{~V}$ 的体系一个时 刻粒子模拟坐标快照. 图中展示模拟系统几何构成、水 分子的瞬时坐标以及电极板铂原子的瞬态电荷. 金属铂 电极板表面为面心立方密堆结构的(111)表面, 我们用 色谱颜色变化标注的电极原子所带电荷的数值, 色带中 间蓝色代表负电，色带中间红色代表正电，色带两端绿 色和白色代表低于和高于中值的电荷数值. 电极原子色 彩变化可以更加直观展示恒定电势约束条件下电极板 电荷与附近极性液体的热毛细涨落以及密度涨落之间 的库伦关联. 虽然本论文没有深入分析水表面的热毛细 波涨落行为, 但是从坐标快照以及时间演化的坐标轨迹 中很容易看出水的表面位置的起伏和波动, 明确具有的 粗鋉类型界面结构.

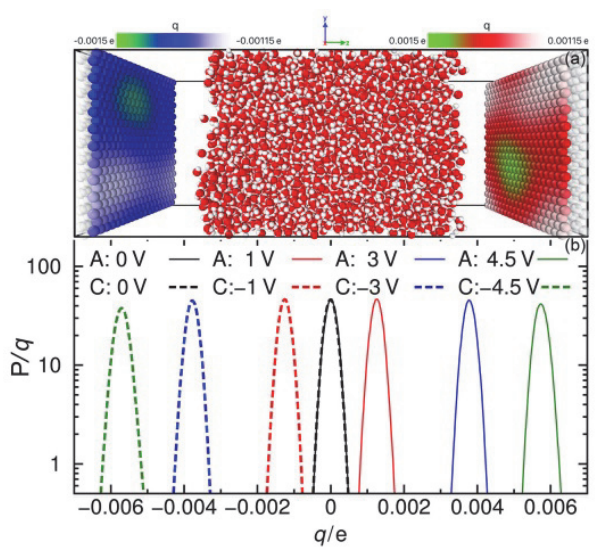

图 1 (a)恒定电势分子动力学模拟坐标快照, 两金属铂电极板电势 2 $V$, 温度 300 K. (b) 4 组不同的施加电势组合下, 阴阳电极板表面原子 所带电荷的概率密度. 纵坐标为对数标度

Figure 1 (a) Constant potential method MD simulation snapshot. The simulation box consists of a water slab between two electrodes maintained at constant potential difference $2 \mathrm{~V}$, temperature $300 \mathrm{~K}$. The color code on the FCC electrode Pt atoms indicates instantaneous charge, with central palette color-blue corresponding to negative charge and red corresponding to positive charge. In the liquid water region, the red spheres represent oxygen and the smaller white spheres represent hydrogen. (b) Log-linear plot of the probability distribution of electrode atom charges for the surface layers of electrodes at four different values of the applied electrode potential differences. Dashed lines correspond to the results at cathode and the solid lines correspond to the results at anode

图 1(b)中画出了电极板表面层原子所带电荷数值分 布的时间平均统计概率，不同颜色和线形代表 4 组不同 的施加电势组合结果. $0 \mathrm{~V}$ 电势差体系中两个电极板电 荷分布概率呈现正态分布并几乎完全重合，原子上带电 量数值分布在 $-0.0005 \mathrm{e}$ 到 $0.0005 \mathrm{e}$ 区间. 电势差变大后, 极板表面原子所带电荷的分布概率也相应发生了明显 的变化, 阴极极板电荷越来越多变为负值, 阳极极板电 荷越来越多变为正值. 与此同时，分布概率形状几乎保 持不变仍可较好的用高斯函数描述. 在前人应用恒定电 势方法模拟水/阴极界面的研究中，由于同一个水分子 
上两个氢氧键均有可能与阴极表面接触以及电势增加 伴随水分子转动, 极板电荷分布概率呈现非正态分布 (双峰分布)的情形 ${ }^{[42]}$. 图 1(b)中所有体系具有几乎相同 的高斯分布形式，是因为水表面到电极表面之间的 3 $\mathrm{nm}$ 距离已经远大于极性液体涨落与电极表面充放电之 间的关联长度.

图 $2 \mathrm{a}, 2 \mathrm{~b}$ 中展示出水表面区域平均水分子数密度分 布函数. 零外电场(电势差 $0 \mathrm{~V}$ ) 体系, 水膜内部平均密度 为 $33.1 \mathrm{~nm}^{-3}$, 这一数值与文献中报道的相同温度下 $\mathrm{SPC} / \mathrm{E}$ 水密度一致 ${ }^{[72,79]}$. 两电极板电势差变大, 水膜的 内部区域密度变化微小与线宽相当, 水表面区域水分子 密度变化明显一些, 表面过渡范围随着电势差变大而变 宽，阴极附近水表面更明显些. 随着极板电势差增大， 水表面外侧(汽相侧)的分子数密度增大, 内侧(液相侧) 分子数密度减小, 这与 Warshavsky 等 ${ }^{[80]}$ 通过平均场密 度泛函理论得到的结论相一致, 即垂直极性液体表面外 加电场作用下, 汽相侧密度逐渐随外电场增强而增加, 液相侧则减小. 以误差函数拟合水分子密度分布函数, 可获得 10 90 表面宽度 $w_{1090}$, 即密度分布函数数值从 液相密度的 $10 \%$ 变化到 $90 \%$ 的宽度范围(图 2). 电势差 从 $0 \mathrm{~V}$ 增加到 $9 \mathrm{~V}$, 阴极附近水表面宽度 $w_{1090}^{\mathrm{C}}$ 从 $0.389(7)$ $\mathrm{nm}$ 增加到了 0.467(8) nm, 阳极附近水表面宽度 $w_{1090}^{\mathrm{A}}$ 增 幅小于阴极附近的水表面, 从 $0.388(6) \mathrm{nm}$ 增加到 0.433(12) nm. $w_{1090}$ 数据详见表 1 .

水表面区域偶极矩极化密度分布函数结果在图 $2 \mathrm{c}$, $2 \mathrm{~d}$ 中画出. 与水分子密度分布函数非常不同, 外电场增 强时偶极矩极化密度分布函数发生了强烈变化. $0 \mathrm{~V}$ 电 势差的体系液膜内部没有极化发生, 水表面存在自发极 化. 我们定义正的偶极极化密度对应着水分子偶极矩垂 直水表面, 且氧原子指向阳极. 电势差变大, 越来越多 的内部水分子发生旋转使水分子偶极矩方向与 $z$ 坐标轴 平行, 反映在水膜内部的偶极极化密度增大及水表面极 化分布强烈调整: 阳极附近水表面极化正峰随电势差增 加而增大, 阴极附近的水表面极化负峰范围收缩, 极化 负峰峰谷数值从负数变成了正数, 并伴随外侧演变出一

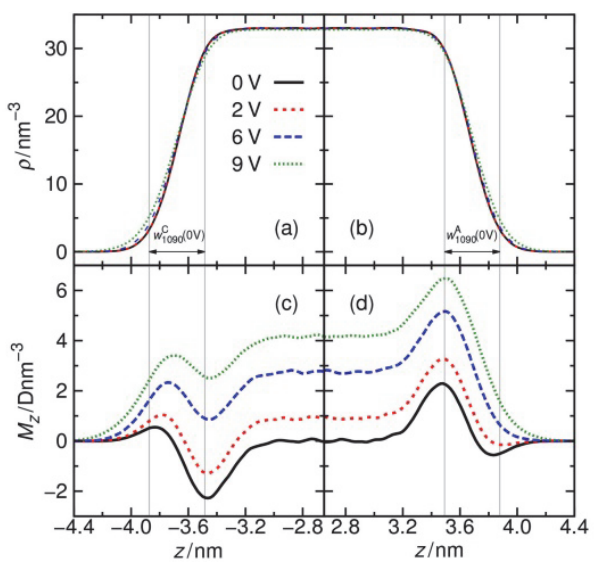

图 2 阴阳两极附近水表面平均分子数密度 $(a, b)$ 和偶极矩极化密度 (c, d) 随恒定电势电极电势差变化. 两平行坚线标出了密度数值从液 相密度的 $10 \%$ 变化到 $90 \%$ 的表面宽度范围

Figure 2 Mean water molecules number density $(a, b)$ and dipole moment ( $z$ component) density (c, d) profiles for water surfaces near cathode and near anode, in the systems with four different values of the applied electrode potential differences. Parallel vertical thin lines locate a distance (10 90 width) over which the density profile changes from $10 \%$ to $90 \%$ of the density in the bulk liquid relative to its value in the vapor as one traverses the surface from the vapor into liquid

个明显的正峰. $M_{Z}(z)$ 统计了单位体积内的平均偶极 矩 $z$ 分量密度, 我们也分析了 $x y$ 分量偶极矩密度分布 $M_{x y}(z)$, 结果表明无论是表面区域还是水膜内部区域, 在任何外电场强度作用下, $M_{x y}$ 均在 0 附近做小幅随机 涨落分布.

应用平均探针法计算获得的体系电势分布函数结 果在图 3 中展示, 图中横坐标范围起于阴极最外层原子 位置 $(z$ : $-7.15 \mathrm{~nm})$, 终于阳极板最外层原子位置 $(z$ : $7.15 \mathrm{~nm}$ ). 图中的 4 条不同线性的线对应着两电极板电 势差为 $0,2,6$ 和 $9 \mathrm{~V}$ 的四组模拟体系的计算结果. 零外 电场( $0 \mathrm{~V}$ 电势差)的体系, 在阴阳两电极区域和真空层 区域的电势数值全部为 0 , 从两边电极分别过渡到两个 水表面处，电势数值在两个表面均出现了下降，在不到 $1 \mathrm{~nm}$ 的空间范围内从 $0 \mathrm{~V}$ 降到 $-0.598 \mathrm{~V}$, 这个电势下降

表 1 从恒定电势分子动力学方法进行的外电场作用下水表面模拟中提取的表面热力学参量. C 代表阴极、 A 代表阳极. $\Delta \phi$ 是两电极板电势差, $w_{1090}$ 是 $10 \sim 90$ 表面宽度, $\chi_{1090}$ 和代表水表面 $10 \%$ 和 $90 \%$ 液相密度位置间电势落差(或表面电势)和偶极极化密度落差. $E_{0}$ 是体系真空层区域匀强电 场强度, $E_{\mathrm{p}}$ 是水膜内部极化电场强度. 括号内数字代表 $95 \%$ 置信度的统计误差

Table 1 Surface thermodynamic parameters extracted from the constant potential method MD simulation of the liquid-vapor water surfaces under different external E-field generated by nearby cathode (C) and anode (A). $\Delta \phi$ is the voltage difference between cathode and anode, $w_{1090}$ is the $10 \sim 90$ width of the water surface, $\chi_{1090}$ and $\mathcal{P}_{1090}$ stand for the water surface potential and surface dipolar polarization density difference, $E_{0}$ is the strength of E-field in the vapor/vacuum region between electrodes and water surfaces, $E_{\mathrm{p}}$ is the strength of E-field within water film due to bulk polarization. Numbers in parentheses are $95 \%$ confidence errors for the last digits shown

\begin{tabular}{ccccccccc}
\hline$\Delta \phi / \mathrm{V}$ & $w_{1090}^{\mathrm{C}} / \mathrm{nm}$ & $w_{1090}^{\mathrm{A}} / \mathrm{nm}$ & $\chi_{1090}^{\mathrm{C}} / \mathrm{V}$ & $\chi_{1090}^{\mathrm{A}} / \mathrm{V}$ & $\begin{array}{c}\mathcal{P}_{1090}^{\mathrm{C}} / \\
\left(\text { Debye } \bullet \mathrm{nm}^{-3}\right)\end{array}$ & $\begin{array}{c}\mathcal{P}_{9010}^{\mathrm{A}} / \\
\left(\text { Debye } \bullet \mathrm{nm}^{-3}\right)\end{array}$ & $E_{0} /\left(\mathrm{V} \cdot \mathrm{nm}^{-1}\right)$ & $E_{\mathrm{p}} /\left(\mathrm{V} \cdot \mathrm{nm}^{-1}\right)$ \\
\hline 0 & $0.389(7)$ & $0.388(6)$ & $0.49(1)$ & $0.49(1)$ & $2.78(3)$ & $2.77(3)$ & 0.00 & 0.000 \\
2 & $0.393(10)$ & $0.385(6)$ & $0.48(2)$ & $0.51(2)$ & $2.10(4)$ & $3.46(5)$ & 0.34 & 0.006 \\
6 & $0.421(7)$ & $0.398(7)$ & $0.43(3)$ & $0.56(1)$ & $0.56(5)$ & $4.58(3)$ & 1.02 & 0.015 \\
9 & $0.467(8)$ & $0.433(12)$ & $0.36(3)$ & $0.62(2)$ & $-0.60(3)$ & $5.31(4)$ & 1.55 & 0.023 \\
\hline
\end{tabular}


的区域与水表面密度从汽相过渡到液相的空间范围几 乎重合. 从表面进入水膜内部, 电势保持在 $-0.598 \mathrm{~V}$ 的恒定数值, 根据前文定义, 本工作零外电场体系的表 面电势 $\chi$ 为 $-0.598 \mathrm{~V}$, Sokhan 和 Tildesley ${ }^{[51]}$ 曾指出过水 表面电势为负值的主要原因来自表面水分子的电四极 矩的贡献. 图 3 中的电势分布函数和表面电势 $\chi$ 结果与 Sokhan 和 Tildesley 计算过的(SPC/E 力场模型描述的) 水表面系统的电势分布函数相似. Sokhan 和 Tildesley 研 究工作中获得的表面电势为 $-0.55 \mathrm{~V}$, 绝对值稍小于本 工作的结果, 数据间的差别可能来自于本工作中应用了 三维周期性 Ewald 求和并进行了周期性盒子的形状修 正，两个工作中界面横截面积也不同，对应着不同的毛 细波涨落厚度也可能对表面电势的数值有部分影响.

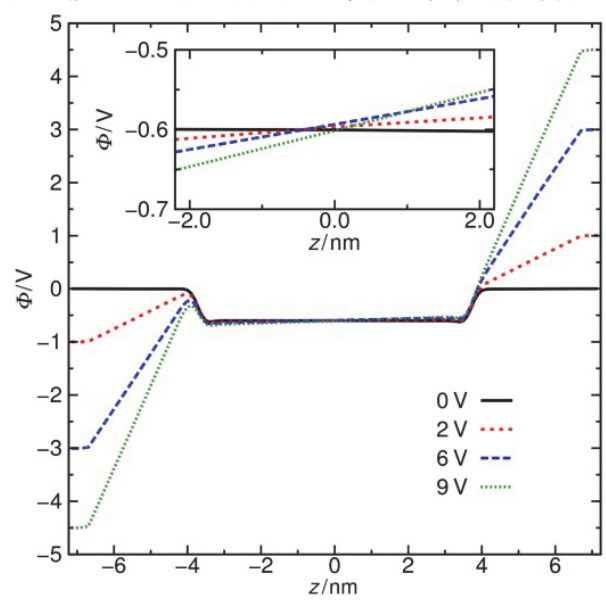

图 3 应用平均探针法计算获得的全局电势分布函数随恒定电势电极 电势差变化结果，插图放大展示液膜内部的电势分布

Figure 3 Electric potential profile from 4 different voltage difference simulations, calculated using the probe and average method. The inset shows selected data portion in the bulk water region

图 3 插图放大展示了水膜内部区域的电势分布. 零 外电场(电势差 $0 \mathrm{~V}$ )的体系水膜内部电势为恒定常数, 这印证了 Wang 等指出的平均探针法相比其他电势计算 方法的收玫性优势, 即通过平均探针法计算获得的电势 分布函数没有因数值积分带来的非物理梯度. 两个电极 板电势差大于 $0 \mathrm{~V}$ 的三组模拟体系, 水膜内部电势空间 分布保持线性变化并出现了明显的梯度, 梯度随电极板 电势差的变大而变大. 此电势梯度变化是水膜中水分子 极化带来的, 分布函数倾斜(电势下降)方向即水膜内部 的平衡电场方向，与电极提供的外电场方向相同.

为了更加清晰的检查水表面局域电势分布随电势 差变化的差异, 图 4(a)和 4(b)中分别放大展示了阴阳两 电极附近两个水表面 $1.3 \mathrm{~nm}$ 局域范围内的电势分布函 数. 阴极附近水表面密度分布函数确定的表面宽度, 近 似在 $z=-3.9 \mathrm{~nm}$ 到 $z=-3.5 \mathrm{~nm}$. 对于 $0 \mathrm{~V}$ 体系, $z<$ $-3.9 \mathrm{~nm}$ 电势趋于 $0 \mathrm{~V}, z>-3.5 \mathrm{~nm}$ 电势趋于内部液体 电势. 相比而言, 电势差为 $2 \mathrm{~V}$ 及以上的体系, 电势从 $z=-3.9 \mathrm{~nm}$ 左右的一个极大点下降, 进入匀强电场区
域，电势沿着电场方向一直下降至阴极设定电势 $(-1 \mathrm{~V}$, $-3 \mathrm{~V},-4.5 \mathrm{~V})$. 在 $-3.9 \mathrm{~nm}>_{z}>-3.5 \mathrm{~nm}$ 区域, 电势 也从极大点下降，不同模拟体系下降区域斜率近乎平 行, 直至 $z=-3.4 \mathrm{~nm}$ 附近的电势低点. 需要指出的是, 这个水表面区域的电势变化趋势和电场方向在整个系 统中很特殊，水表面的分子自发极化电场驱使电势从汽 相到液相下降, 又因阴极附近的水表面液相到汽相的转 换方向与外电场方向相反，此区域的电势最终沿着外电 场相反的方向下降.下降区域电势落差(或水表面电势) 随着电极板间电势差的变大而变小.

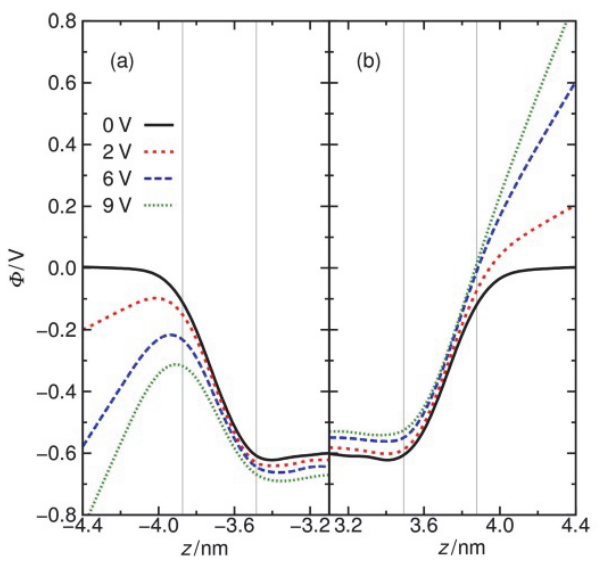

图 4 水表面局域电势分布随电势差变化的差异. (a)阴极附近的水表 面, (b)阳极附近的水表面

Figure 4 Selected surface portion of the electric potential profile from 4 different voltage difference simulations. (a) Water surface near cathode, (b) water surface near anode

相比阴极附近的水表面，阳极附近水表面的电势分 布[图 4(b)]呈现完全不同的变化趋势. 一个明显的区别 是, 电势差 $2 \mathrm{~V}$ 及以上体系在 $z>3.9 \mathrm{~nm}$ 均进入了线性 增加的匀强电场区域，阳极附近的水表面没有形成局域 电势极大值. 在 $z=3.5 \mathrm{~nm}$ 到 $z=3.9 \mathrm{~nm}$ 的表面区域, 电 势从汽相到液相仍快速下降，但与阴极附近水表面不 同，下降方向与外电场方向相同. 电场作用下的两个水 表面电势分布变化的另一个区别也在图 4(b)中呈现: 不 同模拟体系的电势从阳极起下降至 $z=3.4 \mathrm{~nm}$ 左右形成 电势低点, 电势差大的体系的电势低点数值较高, 这和 阴极附近水表面电势低点的情形相反. 这形成了两个水 表面的两个电势低点间保有一个小额的电势差 $(0.1 \mathrm{~V}$ 上 下), 此跨越水膜的小额电势差随电极板间电势差的增 大而变大, 连接其间电势分布函数即展现出如图 3 插图 所示的不同梯度, 具体对应着极化电场强度 $E_{\mathrm{p}}$, 在图 5 和表 1 中具体展示.

外电场强增强对偶极矩极化密度分布函数 $(z$ 分量) 的改变要明显强于对电势分布函数的改变. 两个电极板 间电势差增大时，电场驱使越来越多的水分子调整其偶 极矩朝向, 阳极附近水表面外层水分子和阴极附近水表 面内层水分子在外电场的作用下出现大量的集体转向. 


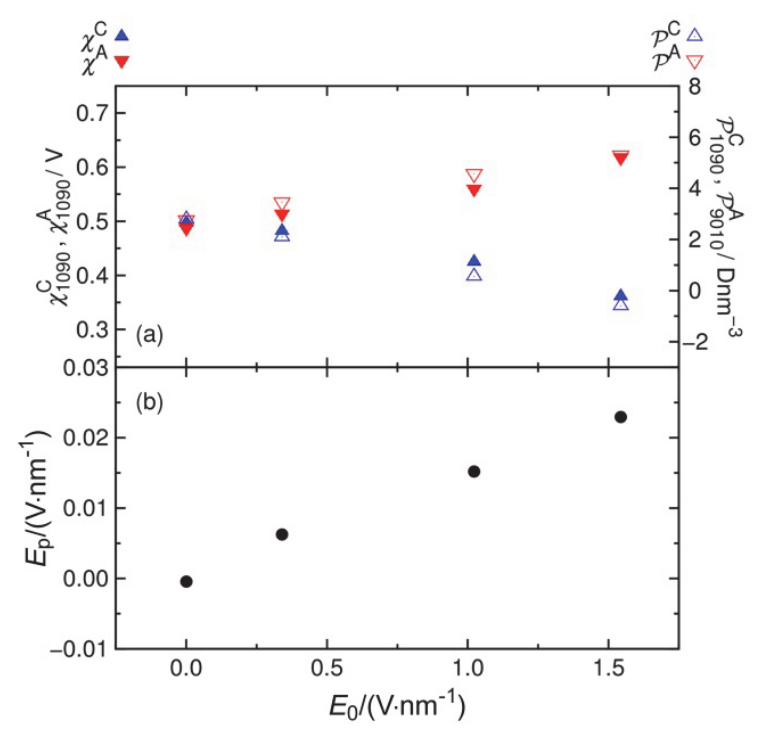

图 5 (a)水表面电势 $\chi_{1090}^{\mathrm{C}} 、 \chi_{1090}^{\mathrm{A}}$ 和水表面偶极极化密度落差 $\mathcal{P}_{1090}^{\mathrm{C}}$ 、 $\mathcal{P}_{9010}^{\mathrm{A}}$ 随外电场强度的变化关系呈现非对称性. (b)水膜内部区域极化 电场强度 $E_{\mathrm{p}}$ 随外电场强度 $E_{0}$ 的变化关系

Figure 5 (a) Water surface potential $\left(\chi_{1090}^{\mathrm{C}}, \chi_{1090}^{\mathrm{A}}\right)$ and water surface dipolar polarization density difference $\left(\mathcal{P}_{1090}^{\mathrm{C}}, \mathcal{P}_{9010}^{\mathrm{A}}\right)$ as the function of $E_{0}$. (b) Strength of E-field within water film due to bulk polarization, $E_{\mathrm{p}}$, as the function of $E_{0}$

水表面氢键网络结构也相应的调整 ${ }^{[34]}$ : 一方面, 表面外 层水分子中的外露氢原子, 在电场作用下向块体水接 近，拓宽氢键网络分布; 另一方面，表面外层水分子中 氧原子直接在外电场作用下接近内层水分子中旋转外 露的氢原子, 拓宽氢键网络分布. 外电场下表面水分子 氢键网络结构的调整或表面偶极极化紧密关联着水的 表面电势分布函数的变化, 具体表现为电势分布函数在 水表面区域随外电场增大的调整(例如, $-3.9 \mathrm{~nm}<z<$ $-3.5 \mathrm{~nm}$ 区域电势分布的下移, $3.5 \mathrm{~nm}<z<3.9 \mathrm{~nm}$ 区域 电势分布的上移), 值得注意的是, 上下平移调整的量 值相对各个体系表面电势(见表 1)的绝对值要小(最多占 $20 \%$ ). 因此, 外电场变强对水表面电势的改变源于水表 面偶极矩极化密度的大幅调整, 但偶极极化调整带给表 面电势的改变量小于表面电势本身量值. 我们在外电场 下水表面电势变化的分析发现支持 Sokhan 和 Tildesley ${ }^{[51]}$ 曾提出的水表面的表面电势中偶极矩仅做次 要贡献的研究认识.

电极板区域电势分布严格与恒定电势方法设定电 势值相等. 电势从阴极/阳极板分别向附近水表面以线 性增加/减小方式变化, 即以匀强电场过渡两个真空层 区域, 即上文中多次提到的外电场. 表 1 列出了所有电 极电势差模拟系统下的真空层电场强度 $E_{0}$. Skinner等的 实验研究比较了强度为 $10^{6}$ 和 $10^{9} \mathrm{~V} / \mathrm{m}$ 的外电场作用下 水的极化行为, 发现 $10^{9} \mathrm{~V} / \mathrm{m}$ 的外电场强度让液桥水的 块体部分极化而 $10^{6} \mathrm{~V} / \mathrm{m}$ 强度的电场却无法极化块体 水. 表 1 所示, 电势差 $6 \mathrm{~V}$ 的体系真空层区域匀强电场 强度 $E_{0}$ 在 $1 \mathrm{~V} / \mathrm{nm}$ (也即 $10^{9} \mathrm{~V} / \mathrm{m}$ ) 左右, 水膜内部确实发
生了极化. 另外, $2 \mathrm{~V}$ 体系水膜内部非 0 的偶极矩极化密 度与 $0.006 \mathrm{~V} / \mathrm{nm}$ 的内部极化电场强度, 说明 $E_{0}$ 在 $10^{8}$ $\mathrm{V} / \mathrm{m}$ 的量级时, 水膜内部块体部分也被极化. 块体水的 极化是否在 0 到 $2 \mathrm{~V}$ 之间一个特定的外电场强度下突变 发生, 还是极化随着外电场增强而连续增加, 还需要近 一步探索. Shi 等 ${ }^{[32]}$ 论文中曾用恒定电场方法研究过外 电场为 $1 \mathrm{~V} / \mathrm{nm}$ 作用下水表面偶极矩极化密度分布函数. 由于恒定电场方法使整个模拟系统不论表面或水膜内 部都保有恒定电场, 诸多物理细节如电场在水表面因介 电极化被弱化甚至被倒转方向等微观现象不能被准确 重现, 因此即使本研究中应用恒定电极电势差生成真空 层外电场强度与 Shi 等工作中强度相当的外电场, 水表 面和内部的偶极矩极化密度分布函数、电场和电势分布 函数均因施加电场的模拟方法不同而呈现差异.

上文中已经计算了本研究中 $0 \mathrm{~V}$ 电势差系统的表面 电势为 $-0.598 \mathrm{~V}$, 定义 (详见参考论文 ${ }^{[52]}$ 及其中引文)为 液相电势减去汽相电势. 外电场不为零情况下, 水表面 区域的电势分布函数变得更加复杂, 液相和汽相区域并 无恒定电势分布区域. 为了获得定量比较, 我们定义密 度分布函数 $10 \%$ 液相密度位置到 $90 \%$ 液相密度位置之 间对应的电势数值之差为表面电势, 计为 $\chi_{1090}^{\mathrm{C}}$ 和 $\chi_{1090}^{\mathrm{A}}$, C 代表阴极附近的水表面, A 代表阳极附近的水表面. 不同电势差模拟体系, 两个水表面的 $\chi_{1090}^{\mathrm{C}}$ 和 $\chi_{1090}^{\mathrm{A}}$ 结果 列在了表 1 中. 图 5(a)画出了水表面电势随外电场强度 变化结果, 随着外电场增强, $\chi_{1090}^{\mathrm{A}}$ 从 0.49(1) V 增加到了 $0.62(3) \mathrm{V}, \chi_{1090}^{\mathrm{C}}$ 从 $0.49(1) \mathrm{V}$ 减小到了 0.36(3) V. 我们发 现电场调控的水表面电势的非对称性行为, 与水表面 $10 \%$ 和 $90 \%$ 液相密度位置间偶极极化密度的落差(见表 1 中 $\mathcal{P}_{1090}^{\mathrm{C}}$ 和 $\left.\mathcal{P}_{9010}^{\mathrm{A}}\right)$ 变化趋势相似. 这意味着在温度不变的 条件下, 表面偶极极化的程度与非对称性是水表面电势 随外电场非对称性变化的主要因素. 曾有模拟计算定量 比较过实验估测的水表面电势随温度变化的梯度系数 $\mathrm{d} \chi / \mathrm{d} T^{[51,52,54]}$, 但从未有水表面电势随外电场强度变化 的结果被报道过. 表 1 中还列出了水膜内部区域(受表 面极化屏蔽)的电场强度 $E_{\mathrm{p}}$, 并在图 5(b)中画出了 $E_{\mathrm{p}}$ 与 外电场强度的 $E_{0}$ 单调递增的变化关系.

\section{4 结论}

水的表面电势具有重要研究价值, 但实验上直接测 量极其困难, 仅有少数研究测量过零外电场下水表面电 势及其随温度的变化. 本论文提出一套基于平衡态恒定 电势分子动力学的模拟一分析-计算方法, 可实现将金属 电极产生的电场作用于附近的水表面，并以平均探针电 势计算方法测量空间电势分布. 我们应用此套方法首次 计算了不同电极施加电势下 $\mathrm{SPC} / \mathrm{E}$ 模型描述的水表面 的空间电势分布函数, 并测量出阴极和阳极附近水表面 的表面电势与外电场的变化关系. 我们发现阳极附近的 水表面电势随外电场增大而变大, 阴极附近的水表面电 
势则随外电场增大而变小. 通过对比两个水表面的电 势、数密度和偶极矩极化密度分布函数, 我们提出电场 调控水表面电势的非对称性, 源自于不同电极附近的水 表面区域在外电场作用下以不同方式偏离自发极化，以 及强电场作用下液体水膜的整体极化. 在此非对称性的 研究发现基础上再引入温度变化, 水表面电势的变化规 律将更加丰富, 这也意味着更多的水表面性质调控可 能.

恒定电极电势分子动力学方法相较其他施加电场 方法(电极恒定电荷法或恒定电场法)更接近实验物理真 实情形, Wang 等 ${ }^{[73]}$ 就曾指出过电极恒定电荷法会导致 电势偏差. 另外, 本论文使用的平均探针电势计算方法 相较传统基于泊松方程的计算方法也拥有诸多优势. 因 此, 我们认为恒定电极电势分子动力学方法结合平均探 针电势计算方法, 是至今探索表面电势的最具竞争力的 理论研究方法. 应用本套方法能够为水表面科学提供重 要数据, 如从表面电势进而获得表面电化学势, 将可获 得直接与和频光谱实验结果相对比的理论数据 ${ }^{[81]}$. 应 用本套方法, 还可获得外电场作用下水表面电势与表面 偶极极化密度数据, 再近一步联系表面介电性值和表面 张力数据的话, 将有机会澄清臭名昭著的关于电场下水 表面张力变化规律的长期争议 ${ }^{[22]}$.

本论文所发展模拟与计算方法适用于各种水模型, 包括能够模拟氢键网络畸变的可极化水分子模型等, 得 到科学价值更高的表面电势数据. 本模拟技术同样适用 于其它极性分子液体体系. 电四极矩对水表面电势的贡 献 $^{[51]}$ 在外电场下的变化以及使用基于第一性原理分子 动力学模拟技术 ${ }^{[52]}$ 从电子云分布联系水表面电势变化 规律本质，将是我们在本文之后的探索方向.

\section{References}

[1] Bateni, A.; Susnar, S. S.; Amirfazli, A.; Neumann, A. W. Langmuir 2004, 20, 7589.

[2] Bateni, A.; Laughton, S. J.; Tavana, H.; Susnar, S. S.; Amirfazli, A.; Neumann, A. Colloid Interface Sci. 2005, 283, 215.

[3] Eggers, J.; Villermaux, E. Rep. Prog. Phys. 2008, 71, 036601.

[4] Yan, J. Y.; Patey, G. N. J. Phys. Chem. Lett. 2011, 2, 2555.

[5] Yan, J. Y.; Patey, G. N. J. Phys. Chem. A 2012, 116, 7057.

[6] Yan, J. Y.; Patey, G. N. J. Chem. Phys. 2013, 139, 144501.

[7] Yan, J.; Overduin, S. D.; Patey, G. N. J. Chem. Phys. 2014, 141, 074501 .

[8] Zhang, Z. S.; Liu, X. Y. Chem. Soc. Rev. 2018, 47, 7116.

[9] Dash, J. G.; Rempel, A. W.; Wettlaufer, J. S. Rev. Mod. Phys. 2006, 78,3 .

[10] Qiu, H.; Guo, W. L. Phys. Rev. Lett. 2013, 110, 195701.

[11] Mei, F.; Zhou, X. Y.; Kou, J. L.; Wu, F. M.; Wang, C. L.; Lu, H. J. J. Chem. Phys. 2015, 142, 134704.

[12] Zangi, R.; Mark, A. E. J. Chem. Phys. 2004, 120, 7123.

[13] Choi, E. M.; Yoon, Y. H.; Lee, S.; Kang, H. Phys. Rev. Lett. 2005, 95, 085701 .

[14] Ehre, D.; Lavert, E.; Lahav, M.; Lubomirsky, L. Science 2010, 327, 672.

[15] Carpenter, K.; Bahadur, V. Langmuir 2015, 31, 2243.

[16] Nandi, P. K.; Burnham, C. J.; English, N. J. J. Chem. Phys. 2018, 148, 044503 .

[17] Zaragoza, A.; Espinosa, J. R.; Ramos, R.; Cobos, J. A.; Aragones, J. L.; Vega, C.; Sanz, E.; Ramírez, J.; Valeriani, C. J. Phys.: Condens. Mat. 2018, 30, 174002.
[18] Fernández, M. S.; Peeters, F. M.; Neek-Amal, M. Phys. Rev. B 2016, 94, 045436.

[19] Vorob'ev, V. S.; Malyshenko, S. P. Phys. Rev. Lett. 2006, 96, 075701.

[20] Maerzke, K. A.; Siepmann, J. I. J. Phys. Chem. B 2010, 114, 4261.

[21] Aragones, J. L.; MacDowell, L. G.; Siepmann, J. I.; Vega1, C. Phys. Rev. Lett. 2011, 107, 155702.

[22] Skinnera, L. B.; Benmorea, C. J.; Shyama, B.; J. K. R. Webera, J. K. R; Pariseb, J. B. Proc. Nat. Acad. Sci. U. S. A. 2012, 109, 16463.

[23] Saitta, A. M.; Saija, F.; Giaquinta, P. V. Phys. Rev. Lett. 2012, 108 , 207801.

[24] Futera, Z.; English, N. J. J. Chem. Phys. 2017, 147, 031102.

[25] Warshavsky, V. B.; Bykov, T. V.; Zeng, X. C. J. Chem. Phys. 2001, $114,1$.

[26] Han, G. Z.; Meng, J. J. Continuum Mech. Thermodyn. 2018, 30, 817.

[27] Hayes, C. F. J. Phys. Chem. 1975, 79, 16.

[28] Pethica, B. A. Langmuir 1998, 14, 3115.

[29] Sato, M.; Kudo, N.; Saito, N. IEEE Transactions on Industry Applications 1998, 34, 2.

[30] Vega, C.; Abascal, J. L. F. Phys. Chem. Chem. Phys. 2011, 13, 19663.

[31] Moore, S. G.; Stevens, M. J.; Grest, G. S. Phys. Rev. E 2015, 91, 022309.

[32] Shi, B.; Agnihotri, M. V.; Chen, S. H.; Black, R.; Singer, S. J. J. Chem. Phys. 2016, 144, 164702.

[33] Koski, J. P.; Moore, S. G.; Grest, G. S.; Stevens, M. J. Phys. Rev. E 2017, 96, 063106.

[34] Nikzad, M.; Azimian, A. R.; Rezaei, M.; Nikzad, S. J. Chem. Phys. 2017, 147, 204701

[35] Jackson, J. D. Classical Electrodynamics, 3rd ed., Wiley, Hoboken, NJ, 1999.

[36] Griffiths, D. J. Introduction to Electrodynamics, 3rd ed.: Prentice-Hall, Upper Saddle River, NJ, 1999.

[37] Fumagalli, L.; Esfandiar, A.; Fabregas, R.; Hu, S.; Ares, P.; Janardanan1, A.; Yang, Q.; Radha, B.; Taniguchi, T.; Watanabe, K.; Gomila, G.; Novoselov, K. S.; Geim, A. K. Science 2018, 360, 1339.

[38] Willard, A. P.; Reed, S. K.; Madden, P. A.; Chandler, D. Faraday Discuss. 2009, 141, 423.

[39] Vatamanu, J.; Borodin, O.; Smith, G. D. J. Am. Chem. Soc. 2010, 132,14825

[40] Merlet, C.; Salanne, M.; Rotenberg, B.; Madden, P. A. J. Phys. Chem. C 2011, 115, 16613.

[41] Merlet, C.; Rotenberg, B.; Madden, P. A.; Taberna, P.-L.; Simon, P.; Gogotsi, Y.; Salanne, M. Nat. Mater. 2012, 11, 306.

[42] Limmer, D. T.; Merlet, C.; Salanne, M.; Chandler, D.; Madden, P. A.; van Roij, P.; Rotenberg, B. Phys. Rev. Lett. 2013, 111, 106102.

[43] Limmer, D. T.; Willard, A. P.; Madden, P.; Chandler, D. Proc. Nat. Acad. Sci. U. S. A. 2013, 110, 4200.

[44] Vatamanu, J.; Vatamanu, M.; Bedrov, D. ACS Nano 2015, 9, 5999.

[45] Vatamanu, J.; Bedrov, D. J. Phys. Chem. Lett. 2015, 6, 3594.

[46] Limmer, D. T.; Willard, A. P.; Madden, P. A.; Chandler, D. J. Phys. Chem. C 2015, 119, 24016.

[47] Parsons, R. Modern Aspects of Electrochemistry, Vol. 1, Ed.: Bokris, J. O.-M. London, Butterworths, 1954.

[48] Matsumoto, M.; Kataoka, Y. J. Chem. Phys. 1988, 88, 3233.

[49] Brodskaya, E. N.; Zakharov, V. V. J. Chem. Phys. 1995, 2, 4595.

[50] Wilson, M. A.; Pohorille, A.; Pratt, L. R. J. Chem. Phys. 1988, 88, 3281.

[51] Sokhan, V. P.; Tildesley, D. J. Mol. Phys. 1997, 92, 625.

[52] Kathmann, S. M.; Kuo, I. W.; Mundy, C. J. J. Am. Chem. Soc. 2008, $130,16556$.

[53] Harder, E.; Roux, B. J. Chem. Phys. 2008, 129, 234706.

[54] Randles, J. E. B. Phys. Chem. Liq. 1977, 7, 107.

[55] Pratt, L. R. J. Phys. Chem. 1992, 96, 25.

[56] Barraclough, C. G.; McTigue, P. T.; Ng, Y. L. J. Electroanal. Chem. $1992,329,9$.

[57] Parfenyuk, V. I. Colloid J. 2002, 64, 588.

[58] Yang, L.; Fishbine, B. H.; Migliori, A.; Pratt, L. R. J. Am. Chem. Soc. 2009, 131, 12373.

[59] Yang, L.; Fishbine, B. H.; Migliori, A.; Pratt, L. R. J. Chem. Phys. 2010, 132, 044701.

[60] Shim, Y.; Kim, H. J.; Jung, Y. Faraday Discuss. 2012, 154, 249.

[61] Feng, G.; Cummings, P. T. J. Phys. Chem. Lett. 2011, 2, 2859.

[62] Feng, G.; Li, S.; Atchison, J. S.; Presser, V.; Cummings, P. T. J. Phys. Chem. C 2013, 117, 9178 
[63] Reed, S. K.; Lanning, O. J.; Madden, P. A. J. Chem. Phys. 2007, 126,084704

[64] Reed, S. K.; Madden, P. A.; Papadopoulos, A. J. Chem. Phys. 2008, 128,124701

[65] Gingrich, T. R.; Wilson, M. Chem. Phys. Lett. 2010, 500, 178.

[66] Wang, Z. X.; Yang, Y.; Olmsted, D. L.; Asta, M. Laird, B. B. J. Chem. Phys. 2014, 141, 184102.

[67] Doppenschmidt, A.; Butt, H.-J. Langmuir 2000, 16, 6709.

[68] Pickering, I.; Paleico, M.; Sirkin, Y. A. P.; Scherlis, D. A.; Factorovich, M. H. J. Phys. Chem. B 2018, 122, 4880.

[69] Berendsen, H. J. C.; Grigera, J. R.; Straatsma, T. P. J. Phys. Chem. 1987, 91, 6269 .

[70] Yeh, I. C.; Berkowitz, M. J. Chem. Phys. 1999, 111, 3155.

[71] Ciccotti, G.; Ryckaert, J. P. Comput. Phys. Rep. 1986, 4, 346.

[72] Alejandre, J.; Chapela, D. J. T. A. J. Chem. Phys. 1995, 120, 15.
[73] Wang, Z. X.; Olmsted, D. L.; Asta, M.; Laird, B. B. J. Phys. Condens. Matter 2016, 28, 464006.

[74] Smith, G., Numerical Solution of Partial Differential Equations: Finite Difference Methods, Oxford, Clarendon, 1985.

[75] Sachs, J. N.; Crozier, P. S.; Woolf, T. B. J. Chem. Phys. 2004, 121, 10847.

[76] Li, S.; Feng, G.; Cummings, P. T. J. Phys. Condens. Matter 2014, $26,284106$.

[77] Skollermo, G. Math. Comput. 1975, 29, 697.

[78] Yang, Y.; Laird, B. B. J. Phys. Chem. B 2014, 118, 8373.

[79] Reynolds, W., Thermodynamic Properties in SI: Graphs, Tables, and Computational Equations for Forty Substances, Stanford, CA, Dept. of Mechanical Engineering, Stanford University, 1979.

[80] Warshavsky, V.; Zeng, X. C. Phy. Rev. E 2003, 68, 051203

[81] Richmond, G. L. Chem. Rev. 2002, 102, 2693. 